Journal de la Société des Océanistes

\section{Journal de la Société des Océanistes}

140 | janvier-juin 2015

Intégration régionale des territoires français dans le Pacifique Sud

\title{
Compte rendu de Tabiteuea Kiribati, de Guigone
}

CAMUS

\section{Gilles Bounoure}

\section{(2) OpenEdition Journals}

Édition électronique

URL : http://journals.openedition.org/jso/7307

DOI : $10.4000 /$ jso.7307

ISSN : $1760-7256$

Éditeur

Société des océanistes

\section{Édition imprimée}

Date de publication : 15 juin 2015

Pagination : 156-159

ISBN : 9782854301250

ISSN : 0300-953x

Référence électronique

Gilles Bounoure, "Compte rendu de Tabiteuea Kiribati, de Guigone camus », Journal de la Société des Océanistes [En ligne], 140 | janvier-juin 2015, mis en ligne le 06 juillet 2015, consulté le 24 septembre 2020. URL : http://journals.openedition.org/jso/7307 ; DOI : https://doi.org/10.4000/jso.7307

Ce document a été généré automatiquement le 24 septembre 2020.

(c) Tous droits réservés 


\title{
Compte rendu de Tabiteuea Kiribati, de Guigone CAMUS
}

\author{
Gilles Bounoure
}

\section{RÉFÉRENCE}

CAMUS Guigone, 2014. Tabiteuea Kiribati, Paris-Genève, Hazan-Fondation culturelle Musée Barbier-Mueller, 184 p., préface de Jean-Paul Latouche, postface de Monique Jeudy-Ballini, bibliogr., glossaire « aide-mémoire », environ 150 ill. noir et blanc et couleur. 


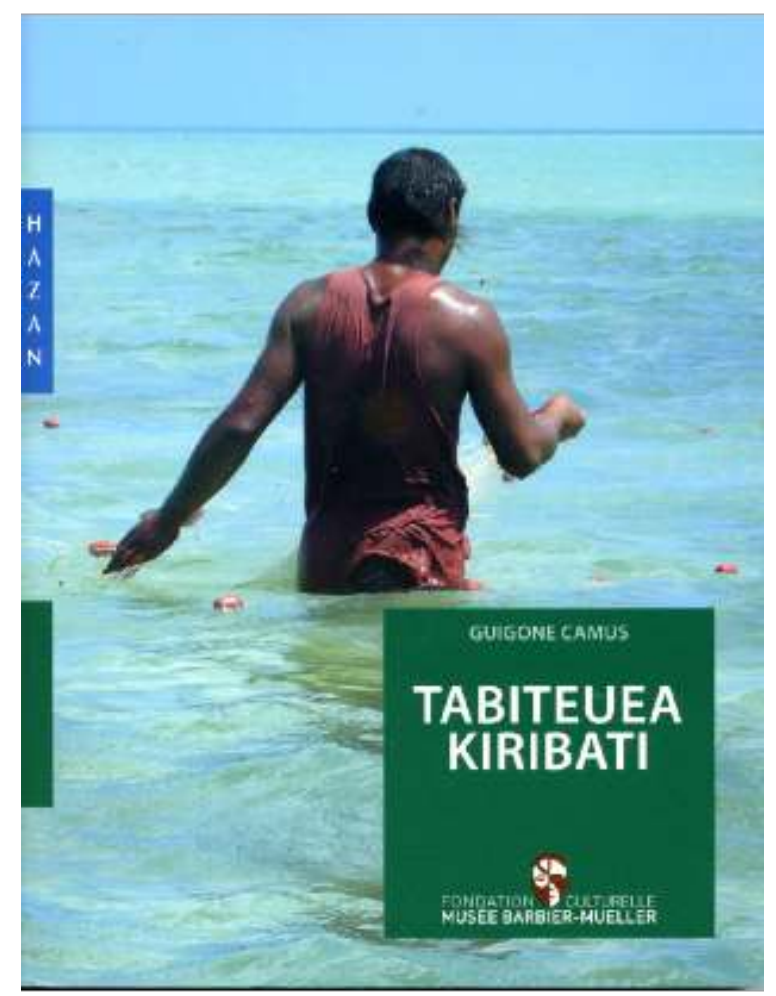

1 Les lecteurs qui entament les livres par la fin - comme il arrive souvent pour les publications à caractère scientifique, par «réflexe induit» ainsi que disent les spécialistes de physiologie animale - s'étonneront, dans les notes et la bibliographie de ce volume, de l'omission de toute référence à Gerd Koch, qui avec son épouse Sigrid rapporta de son séjour dans l'archipel gilbertin, entre août 1964 et mars 1965, plusieurs centaines d'objets pour le musée de Berlin, un ouvrage vite devenu classique (Koch 1965), divers articles importants, plus de mille sept cents photos et soixante-dix films ethnographiques sélectionnés aussitôt par l'Encyclopaedia Cinematographica, dont quatorze tournés à Tabiteuea même, avec pour chacun d'eux l'édition d'un fascicule enrichissant les données du livre précité (Melk-Koch, 2014 : 62). Il est à craindre que l'explication ne réside une fois de plus dans le « germanicum est, non legitur ", posture par laquelle beaucoup de spécialistes actuels se privent délibérément des apports de la science en langue allemande, à la façon des clercs de l'Occident médiéval se flattant d'ignorer le grec. L'ouvrage de G. Camus mentionne certes deux sources en allemand (n'offrant que quelques phrases d'intérêt anecdotique pour le sujet envisagé, par exemple p. 93) mais laisse de côté nombre de publications importantes signalées et exploitées par G. Koch, dont le travail, à cet égard comme à d'autres plus décisifs encore, reste non remplacé.

2 Mais il faut souligner a contrario que ce volume, dénué d'ambition encyclopédique, offre à un prix particulièrement abordable pour le "grand public » un texte rédigé en français, très généreusement illustré de photos, quelquefois en double page, issu des travaux récents d'une ethnologue en cours de doctorat, sur une île et un archipel de Micronésie n'ayant donné lieu jusqu'à présent qu'à un nombre infime de publications francophones. On le présenterait volontiers comme une utile introduction à l'étude des subtilités d'une société traditionnelle encore méconnue, même de beaucoup de spécialistes de l'Océanie. Comme y insistent justement J.-P. Latouche et M. Jeudy-Ballini en tête et en fin de volume, cette publication, avec son « heureuse manière de légitimer 
et conserver intact le souci de connaître ", constitue aussi un encouragement notable aux recherches de l'auteure et aux jeunes ethnologues présents ou à venir, ainsi qu'à celles et à ceux qui les accueillent et les informent. Il est heureux qu'ait été éditée simultanément une version anglaise (disponible auprès de la fondation Barbier-Mueller et de l'éditeur), plus aisément accessible aux insulaires du Pacifique.

L'ouvrage est ordonné en quatre chapitres, "Tabiteuea: une île, une histoire» (pp. 18-75), « Tabiteuea, un foyer mythologique génésiaque » (pp. 76-89), « la maneaba, une architecture du social » (pp. 90-123), et « The News from Tanaeang by Ntongantonga (1948), une épopée de la fondation de Tanaeang » (pp. 124-159). Le deuxième et le quatrième intéressent ce que J.-P. Latouche (1984) a intitulé "la mythistoire Tungaru» et s'appuient sur des documents restés inédits, retrouvés par G. Camus dans divers fonds d'archives, notamment les papiers que conserve le Bishop Museum de l'ethnologue et ethnobotaniste Katharine Luomala, présente à Tabiteuea durant une moitié de l'année 1948 (l'auteure lui rend hommage pp. 24-25). Si leur lecture présente un grand intérêt, on sait que l'étude de tels récits, dont la publication s'est accélérée ces dernières décennies, tend à devenir de plus en plus délicate ou épineuse, du fait des divergences qu'ils offrent d'un atoll à l'autre, voire entre îlots ou clans voisins, non sans enjeux politiques et fonciers dans ces derniers cas. Dans son analyse du texte de Ntongantonga dont elle reproduit aussi le texte gilbertais, G. Camus suggère à plusieurs reprises ces difficultés, mais se concentre avec raison sur la « critique interne » de cette épopée en réduction.

4 Le troisième chapitre consacré aux halles de réunion (maneaba) propres aux i-Kiribati (avec beaucoup de parallèles en Océanie) synthétise les observations de l'auteure et de divers chercheurs l'ayant précédée, spécialement celles de J.-P. Latouche $(1984,1994)$ et de Petra Autio (2010, excellente thèse disponible sur internet). Les deux chapitres précités en montrent l'importance historique, celui-ci en étudie l'architecture et le fonctionnement protocolaires, et ce qu'ils impliquent de prérogatives politiques et sociales, formant encore l'objet de "débats virulents » où s'exprime à l'occasion tel ou tel «ressentiment» tiré de l'histoire récente ou ancienne (pp. 117-118). Par respect pour ses informateurs, l'ethnologue s'est gardée de relater le détail de ces différends, se concentrant sur les "droits et obligations » attachés à la construction et à l'occupation des maneaba qu'elle a pu étudier et s'en tenant à leurs aspects "sociaux", sans référence aux règles de tenure des terres actuellement en vigueur à Kiribati (voir par exemple Atanraoi 1995). Si les piliers de corail supportant les vastes charpentes des maneaba reposent souvent sur des dépôts de fondation, avec "autrefois, crânes d'ancêtres» (p. 101), c'est probablement, ajoutera-t-on ici, que cette architecture prolonge celle de «maisons des esprits » (bata $n$ anti) emportées par la colonisation et la christianisation (Koch, $1965: 149$ ).

5 Le premier chapitre offre de nombreux apports sur l'histoire des "contacts » des Occidentaux avec les insulaires de Tabiteuea, traitant spécialement du " passage de l' U.S. Exploring Expedition» conduite par Wilkes en 1841 (pp. 53-62), avec d'intéressants documents illustrés retrouvés par l'auteure dans les archives navales de Washington et d'autres collections, puis des périodes « 1868-1880, des religions en lutte » et «1892, la colonisation britannique» (pp. 62-69), expliquant avec clarté comment les missionnaires protestants durent céder la place à leurs rivaux catholiques, non sans affrontements sévères entre les insulaires. Les premières pages de cette section sont sans doute les moins réussies de l'ouvrage, en tout cas les moins adaptées à son 
ambition de faire découvrir la vie des habitants actuels de Tabiteuea, justifiant les très nombreuses et belles photos dont l'auteure illustre son propos, au risque d'enjoliver la réalité.

Dans son «esquisse de l'environnement terrestre » (pp. 33-35) G. Camus décrit les « contraintes» imposées aux insulaires par la pauvreté, l'aridité et la salinité des sols, pour souligner «leur habileté à en tirer parti » grâce à des cultures comme celle du cocotier, "principale plante nourricière [...], supportant les sols salins ", comme s'il ne lui arrivait pas de succomber aux effets conjugués de la sécheresse, des tempêtes et de la hausse du niveau des océans. G. Koch $(2003: 36-37)$ a rapporté sa première visite à Aiwa, au centre de Tabiteuea, en 1964,

«die grausamste Eiland das ich je gesehen habe. Das Land ist verbrannt von der Sonne, von vielen Kokospalsmen stehen nur noch die nackten absgestorben Stämme, die Pflanzungsgruben sind im salzigen Wasser ertrunken und unfruchtbar geworden. Stürmisches Meer hat Teile der Insel abgespült und die Gräber aufgewühlt, so dass bleichende Menschenknochen über das Land verstreut sind. Niemand kümmert sich darum... "

7 C'était il y a un demi-siècle, avant les premiers effets notables de changement climatique, et les photos prises alors par Koch permettent aux spécialistes actuels d'étudier l'évolution des traits de côte durant ce laps de temps. L'exposé de G. Camus sur les ressources végétales (pp. 33-40) est évidemment à compléter des données publiées par Koch d'après ses collectes effectuées pour l'herbier du Musée botanique de Berlin (2002:62-94), sa présentation des insulaires, toute d'empathie, reste imprécise on ignore par exemple si Tabiteuea connaît la même "explosion démographique " naguère relevée par Anne Di Piazza (2001: 46) à Nikunau, la même «émigration sans précédent", etc. Surtout, si l'on excepte une formule allusive de J.-P. Latouche sur "l'Océan, dont [Kiribati] tire une bonne partie de ses rares ressources, [devenu] une menace, en raison de notre folie » (p. 9), rien ne signale aux lecteurs de l'ouvrage la préoccupation majeure des habitants actuels de Tabiteuea, que les derniers rapports gouvernementaux disponibles (2012 a et b: 6, voir aussi Klepp, 2013) expriment dans les mêmes termes pour le nord et le sud de l'atoll:

"Coastal erosion is fast becoming the major environmental issue for the islands of Kiribati including Tabiteuea [...]. Many locations on Tabiteuea [...] have been seriously eroded, resulting in the relocation of infrastructure (road, buildings, etc.) or the recurrent high expenditure of maintaining seawalls for protection, "

avec ces détails pour la partie sud:

«Flooding during high tides has been experienced in the village of Tewai, at Buatua and at Taungaeaka. These floods result in saltwater pollution of the freshwater lens which in turn leads to dying fruit trees and bwabwai and brackish well water. Taungaeaka also suffers from erosion starting from where the causeway ends at the village area to nearly halfway through the village. Takuu at the southern end of the island suffers from extreme erosion where rows of trees have fallen with a few left standing on the beach. »

Il n'aurait pas été déplacé, dans un ouvrage principalement destiné au public occidental, de l'informer même brièvement de l'omniprésence et de l'aggravation de cette «menace », due à " notre folie », précisément. 


\section{BIBLIOGRAPHIE}

ATANRAOI Pole, 1995. Customary Land and Development in an Atoll Nation : the case of Kiribati, in Ron Crocombe (ed.), Customary Land Tenure and Sustainable Development. Complementarity or Conflict?, Nouméa-Suva, South Pacific Commission-uSP, pp. 55-73.

AUTIO Petra M., 2010. Hard custom, hard dance. (Un)Differentiation and notions of power in a Tabiteuean community, Southern Kiribati, Helsinki, University of Helsinki Press (en ligne).

DI PIAZZA Anne, 2001. Terre d'abondance ou terre de misère. Représentation de la sécheresse à Nikunau (République de Kiribati, Pacifique central), L'Homme 157, pp. 35-58.

KLEPP Silja, 2013. Kleine Inselstaaten und die Klimabewegung : der Fall Kiribati, in Matthias Dietz et Heiko Garrelts (Hg.), Die Klimabewegungen. Ein Handbuch, Wiesbaden, Springer, pp. 413-428.

косн Gerd, 1965. Materielle Kultur der Gilbert-Inseln, Nonouti, Tabiteuea, Onotoa, Berlin, Museum für Völkerkunde.

-, 2003. Pflanzen für Menschen auf Riffinseln im Pazifik: Niutao + Nonouti, Dresden, Failima.

-, 2003. Man wusste nicht viel voneinander. Ein Ethnologe unterwegs im Pazifik, Dresden, Failima.

LATOUCHE Jean-Paul, 1984. Mythistoire Tungaru. Cosmologies et généalogies aux îles Gilbert, Paris, SELAF.

-, 1994. Conflits et représentation mythique de l'espace, Journal de la Société des Océanistes 98, pp. 43-54.

MELK-КОСн Marion, 2014. Where is the culture?, in Mark Amery et al., Tungaru. The Kiribati project, Auckland, Aukland War Memorial Museum, pp. 59-62.

REPUBLIC OF KIRIBATI ISLAND REPORTS SERIES, 2012. a) 12. Tabiteuea North, b) 13. Tabiteuea South, Bairiki, Office of Te Beretitenti. 